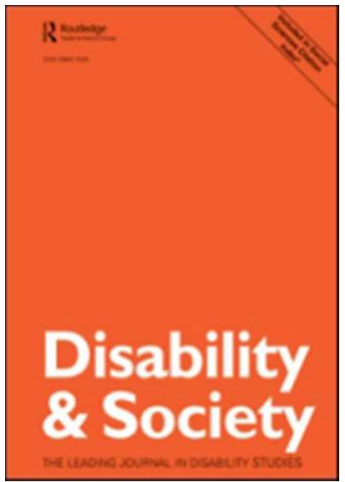

\title{
Perspectives of the National Disability Insurance Scheme: Participants' knowledge and expectations of the Scheme
}

\begin{tabular}{|r|l|}
\hline Journal: & Disability \& Society \\
\hline Manuscript ID & CDSO-2017-0184.R2 \\
\hline Manuscript Type: & Original Article \\
\hline Keywords: & $\begin{array}{l}\text { Self-direction, Participant knowledge, National Disability Insurance } \\
\text { Scheme, Decision-making }\end{array}$ \\
\hline \multirow{2}{*}{ Abstract: } & $\begin{array}{l}\text { Limited research investigates future participants' perspectives of the } \\
\text { National Disability Insurance Scheme - Australia's first national attempt } \\
\text { towards self-directed disability service provision. To identify future } \\
\text { participants' perspectives, seventy people with disability in South-East } \\
\text { Queensland participated in an interview which collected both quantitative } \\
\text { and qualitative data. Over half the participants demonstrated having little } \\
\text { to no knowledge of the NDIS. Participants' expected that: (i) they would } \\
\text { make decisions around organising and paying support and (ii) that their } \\
\text { current service provider would provide the support. Participants also } \\
\text { indicated that training around budgeting and hiring would be essential to } \\
\text { ensure their successfully engagement with the NDIS. The findings are } \\
\text { timely and provide a first-hand account of how people with disability intend } \\
\text { to engage with self-directed support programs. Findings also imply that } \\
\text { targeted information dissemination and participant training and support are } \\
\text { necessary to ensure their successful participation within self-directed } \\
\text { programs. }\end{array}$ \\
\hline
\end{tabular}




\section{Points of Interest}

- Few studies have investigated how people with disability intend to organise their health and social supports within self-directed environments where they are provided direct payments.

- This research found that many people with disability remain unaware of how the National Disability Insurance Scheme (NDIS) - a self-directed approach to disability service provision - will impact the services they receive.

- Future participants' intend that family members will take a central role organising supports, and that service providers will be selected based on familiarity and experiential knowledge.

- Participants' indicated that in order for them to successfully engage with the NDIS they will require training and longstanding support around hiring support staff and budgeting direct payments.

- Using accessible methods to provide NDIS information, and the use of peer-support models, can support people with disability to successfully engage with the NDIS. 


\section{Introduction}

Since July 2016, Australians up to 65 years of age, with permanent and significant disabilities have been able to apply for whole of life disability supports and services. The intent of the National Disability Insurance Scheme Act 2013 (NDIS Act) is to facilitate the full inclusion of people with disability in the mainstream Australian community, and to maximise independent lifestyles for all Australians with disability (Commonwealth of Australia 2013). The NDIS is Australia's first legislation embedding self-directed policy for people with disabilities. Under the NDIS it is expected that individuals will have (i) greater access to resources (including financial resources) and (ii) decision making autonomy to organise their own health and social supports. People with disability will have more flexibility to choose and decide about their disability services and supports: who provides them, how they are designed and supplied, how their resources are used, and how their funding is managed (NDIS Act 2013). The National Disability Insurance Agency (NDIA) is responsible for implementing the NDIS legislation and its rollout across Australia by 2019. NDIS numbers are forecast to increase from just over 30,000 in 2016 to over 450,000 by 2019 (National Commission of Audit). Consequently, it is important to understand future participants' perspectives and expectations of the NDIS.

The NDIS has been trialled in several sites across Australia since July 2013 and is currently in place throughout several parts of the country. In Queensland, the NDIS was introduced throughout parts of the state from July 2016 onwards, and is currently being progressively rolled out throughout the entire state. Concerning the rollout, Townsville, Mackay, and Toowoomba will be in receipt from 1 July 2016 to 30 June 2017, Ipswich, Bundaberg, and Rockhampton will be in receipt from 1 July 2017 to 30 June 2018, and finally Beenleigh, Cairns, Brisbane (North and South), Mayborough, Caboolture/Strathpine, Maroochydore, and Robina will be in receipt from 1 July 2018 to 30 June 2019.It is currently expected that the NDIS will be available across all of Queensland by July 2019. While much of Australia has already shifted to the NDIS, the NDIS is still being introduced in some states (see for example Tasmania).

\section{Self-Directed Policy for People with Disabilities: Research to date}

The self-directed approach to decision making and resource allocation for people with disability has its origins in the 'independent living movement' initiated in the United States of America throughout the 1970s (Gross et al. 2013; Sims and Cabrita Gulyurtlu 2014). As it 
currently stands, self-directed approaches to disability care are generally characterised as health care models where people with disability are provided greater choice and control over their health and social care provision, and this often involves control over financial resources required for their health and social support (Espiner and Hartnett 2012; Gross et al. 2013; Eriksson 2014). Considerable advancement to the self-directed approach to care for people with disability has taken place throughout the last decade (Sims and Cabrita Gulyurtlu 2014), and this is best evidenced by recent legislation (see for example, England's Care and Support (Direct Payments) Regulations 2014, the Social Services and Well-being (Wales) Act 2014, and the Social Care [Self-directed Support] [Scotland] Act 2013).

Given the wide implementation of self-directed support for people with disability, a growing body of research has sought to provide evidence to better inform the approach. Four literature reviews over the last five years have explored the impact of self-direction for people with disability (Harkes 2014; Sims \& Cabrita Gulyurtlu 2014; Crozier et al. 2013; Lakhani, McDonald, and Zeeman 2016). Reviews have generally found limited empirical research exploring the impact of approaches on participants and their families (Harkes et al. 2014b, Sims \& Cabrita Gulyurtlu 2014; Crozier et al. 2013). Findings from the limited research to date suggests that self-directed support has the potential to enable people with disabilities to access health and social support services that they may have not received otherwise (Lakhani, McDonald, and Zeeman 2016), and that this may contribute to their increased quality of life (Harkes, Brown, and Horsburgh 2014; Sims and Cabrita Gulyurtlu, 2014). However, it is also apparent that barriers including inaccessible materials and a lack of professional support in organising resources can potentially prevent the effective engagement of those with disabilities into self-directed models (Sims and Cabrita Gulyurlu, 2014; Lakhani, McDonald, and Zeeman 2016). Considerable knowledge gaps exist about the best way to implement selfdirected services and the impact of these services. So, further research is required to understand the impact of the approach on participants (Harkes, Brown, and Horsburgh 2014; Sims and Cabrita Gulyurtlu, 2014), particularly about how participants intend to organise their supports in a self-directed environment (Lakhani, McDonald, and Zeeman 2016).

\section{The National Disability Insurance Scheme}

The NDIS defines a new era in disability service delivery across all Australian states and territories because it heralds the marketization of disability services delivery in Australia . Several policy tensions are at stake, particularly between the policy promise of 'choice and control' and the legislative caveat of 'reasonable and necessary'. Supports are only funded if 
they are considered 'reasonable and necessary' to help a participant reach their goals, objectives and aspirations; criteria include ensuring that the supports represent "value for money'. (See Foster et al. [2016] and Kendrick, Ward, and Chenoweth. [2017] for discussion on the challenges of equitably implementing the 'reasonable and necessary' proviso within a person-centred framework). However, the pre-eminent challenge is to safely and comprehensively serve the wellbeing of Australians with disability and their families in a competitive, market-driven economy.

This shift in national policy and practice context demands that organisations re-invest in capacity building - for individuals and families, as well as their workforce-knowledge development, and community partnerships to sustain quality client services. Further, it has been suggested that the ambitious goals of the NDIS will only be achieved if this capacity building and investment in families as well the disability service workforce is longstanding over decades (See Kendrick et al. [2017] for six lessons that can affect the success of the NDIS). Specifically, organisations will need to understand how self-direction policy can be implemented and sustained in practice. Published self-directed evaluation research in Australia (see Ottman and Mohebbi [2014]) has identified several barriers to self-directed practices for older Australians. These include lack of client empowerment, clients being inadequately informed about programme options, clients unaware of entitlements, lack of financial transparency, clients not receiving financial statements, limited use of translation services and translated documents, health and psycho-social support, lack of self-confidence, health issues, client/carer preferences, desire for a period of consistency or stability, reluctance to change existing arrangements, lack of time/not wanting extra work or responsibility (carers and clients), and no desire to handle money.

The NDIS is expected to favourably influence the health and wellbeing of people with disability in Australia by ensuring their equitable access to health services (Bonyhady 2014; Russi 2014). However, given the recency of the NDIS, it is difficult to conclude definitively the impact it has on participants. Research is only beginning to explore the perspectives of, and impact of the NDIS on participants, and their family members and/or carers. For example, research undertaken by Ranasinghe et al (2017) investigated the perspectives of parents who organised support and services for their child about NDIS engagement. Participating parents clarified that: (i) registration was not difficult, (ii) receiving funding was not difficult, and (iii) it was easy to correspond with the NDIA. Additionally, the Evaluating the NDIS: Intermediate Report by Mavromaras, Moskos, and Mahuteau (2016) evaluated the 
impact of the NDIS on service users within five trial sites: South Australia, Tasmania, the Hunter region in New South Wales, the Barwon region in Victoria and the Australian Capital Territory. Approximately half the surveyed participants indicated that their level of choice and the quality of their supports improved under the NDIS. They also indicated that it was difficult to establish their NDIS eligibility and that engaging with an advocate with experience working within the NDIS would have favourably supported their engagement. Prospective participants need to be ready for the NDIS if they are to benefit safely and effectively from the promised flexibility of 'choice and control'. Most, if not all, people with disability and their families and carers will need help in taking up the opportunities of the new NDIS way of choosing, managing, and reviewing their supports and services.

Consequently, it is important to understand future participants' perspectives of the NDIS, and how they intend to organise their supports. Research in this area can work to inform service provider practice and the practice of the NDIA.

\section{The Current Study}

It is imperative to establish how people with disability understand the NDIS and intend to organise their supports under the NDIS. This research was conducted as a part of a larger study undertaken throughout May to October 2015. The study -commissioned by a major Australian organisation working with, and providing support to people with disability - aimed to ascertain the supports that their service users currently receive, and what supports service users would require in the future under the NDIS. Given the then-pending roll out of the NDIS throughout Queensland from 2016 to 2019, a component of the study aimed to ascertain service user knowledge of the NDIS, service user perspectives of who would organise their supports under the NDIS, and the types of training that would be required to ensure their successful engagement with the NDIS. This study aimed to answer the following research questions:

1. What do participants know about the NDIS?

2. Who do participants intend to have organise their support under the NDIS?

3. What training and support will participants' require to successfully engage with the NDIS?

\section{Method}


A mixed methods approach was adopted to ascertain research participant knowledge of the NDIS, who they intend to organise their supports with under the NDIS, and the training they would require for their successful engagement with the NDIS. An interview administered cross-sectional survey gathering both qualitative and quantitative data was used. The research methods undertaken as a part of this study were informed by previous research investigating the process of self-direction for people with disability. The researchers' suggestion to directly involve the organisation's clients in the research design was not taken up by the organisation. Instead, the researchers established a process of regular meetings and consultation with frontline and senior staff from the commissioning organisation working directly with people with disability. Previous studies have used qualitative descriptive research designs to identify the perspectives that people with disability and their guardians or support workers have regarding their engagement with self-directed support programs (Dew et al. 2013; Eriksson 2014; F. Mitchell 2012, 2014b). Such research has added new knowledge about the barriers which inhibit and factors which support successful engagement with self-directed policies (F. Mitchell 2014a) and how recipients expect such policies to impact the supports they receive (Espiner and Hartnett 2012). Additionally, quantitative approaches incorporating crosssectional surveys have also been used to establish individuals' satisfaction of self-directed support programs (Heller et al. 2012). Given the success of both quantitative and qualitative approaches to obtain the perspectives of, and outcomes of, self-directed support programs, a mixed methods approach was identified as an appropriate approach for investigating the three research questions underpinning this study.

Previous studies have provided valuable methodological insights particular to their application of a consensus approach whereby interview protocols aim to capture the aggregated opinions of both people with disability and their guardians or support workers as one set of findings (W. Mitchell 2012a; F. Mitchell 2014a, 2014b). In such studies, guardians, family members and/or carers were present to (i) support the communication of people with disability while also (ii) providing the response they felt best reflected the response that their family member with disability would have indicated (ie. when the quality of the communication between the interviewer and person with disability made it difficult to ascertain the latter's response). Thus, these studies have engaged both people with disability and their guardian(s), family member(s), and/or carer(s) in single group interviews, and treated findings cumulatively. This approach was employed as a part of this study. 
Ethics approval for the project was granted by the Griffith University Human Research Ethics Committee (Protocol \#HREC HSV/06/2015) in accordance with the National Health and Medical Research Commission (NHMRC) Human Ethics best practice. Potential participants were identified through a third party consent process as stipulated by the ethics approval and on behalf of the University researchers.

\section{Recruitment and participant details}

Research participants included people with disability and their guardian(s), family member(s) and/or carer(s). Consequently, the term 'research participants' in this paper refers to the individual with disability and their guardian(s), family member(s), and/or carer(s). People with disability were recruited from the commissioning disability service provider's customer database. They had to meet two inclusion criteria. First, they had to reside in the southern district of South-East Queensland, or be located in one regional location outside of this district also included as a part of this research. Second, they had to be of working age i.e., between the ages of 18 and 65 years. Given these criteria, a potential pool of 489 people with disability was identified. The primary disability diagnosis of 414 of these 489 people according to the organisation's database was intellectual disability. Others also had autism, acquired brain injury, psychiatric disability, physical disability, cerebral palsy, neurological disability, vision impairment and hearing impairment.

The organisation facilitated the recruitment process by mailing an invitation to all potential people with disability $(n=489)$ and their families or carers $(n=420)$ on behalf of the researchers. Subsequently, 92 potential research participants consented to be contacted by the researchers, representing a final service user response rate of 19\%. After contacting all 92 potential participants, 70 research participants agreed to be interviewed.

\section{Communication and participation considerations}

Researchers began each interview by reviewing the information mailed out to prior to the interview and clarifying the purpose and goal of the research project with the research participants. Research participants were advised that the research project aimed to understand their knowledge of the NDIS and how they intended to organise their support in the future under the NDIS. Research participants were reminded that they could withdraw from the interview at any time; their responses would not be shared with anyone without their consent; and their commentary would be de-identified in any reports resulting from the research. Researchers reviewed the 'consent to be interviewed' form with the research participants by 
asking if they agreed to each of the check-boxed points on the consent form, and checked each item that the research participants agreed to. The participants were then asked to sign their consent to participate in the research project.

To facilitate better communication between the research interviewers and people with disability, many of whom had significant speech and language difficulties, a customised, 'low-tech' aided communication tool was developed and used specifically for the research (McDonald, Lakhani, and Zeeman, 2016). A Speech Language Therapist provided content and training in this tool for the researchers prior to the interviews. This training included strategies for interacting with people who have complex communication needs (RemingtonGurney 2013; Lakhani et al. 2017), using the Key Word Sign system in common phrases, and adapting the Talking Mat (Murphy and Cameron 2008) for aided language interactions.

\section{Data collection}

The three research questions underpinning this study were answered via the interview protocol as outlined below. Initially the interview protocol included demographic questions. After, the protocol included questions pertaining to the three research questions comprising this study.

\section{Demographic questions}

The protocol included background and demographic questions as well as the EuroQol 5D UK English Version (Cheung et al. 2009). Background and demographic information gathered included: age, gender, length of time receiving services from the organisation, and support services received by the organisation. The EuroQol survey ascertained the person with disability's overall health and wellbeing status across the five domains of mobility, self-care, usual activities, pain/discomfort, and worried/sad. The research study added two other domains: eating/drinking and communication. Research participants were asked to indicate their level of capacity for each domain from three response options for each question. The three response options were scaled so that the first response option was either no difficulty, pain or sadness, the second response option wasmoderate difficulty, pain, or sadness, and the third response option was extreme difficulty, pain or sadness.

\section{Support under the National Disability Insurance Scheme}

Questions were included to answer the three research questions; these are outlined below and aligned with each research aim. 


\section{RQ1: What do research participants know about the NDIS?}

Open-ended questions were used to help answer this research question:

- What do you know about the NDIS?

- Have you been to any NDIS seminars or information workshops?

RQ2: Who do research participants intend to have organise their support under the NDIS?

An open-ended question and a set of questions with multiple response options were included to answer this research question. Research participants were first asked an open ended question - "In the future, who will make most of the decisions about your support?" A detailed multi-response question set was then used to ascertain who would make decisions across a variety of domains. Research participants were requested to indicate, who they intend to provide future support, across the following domains: selecting help, changing help, keeping money, and finding support. Response options for these questions included: me by myself, me and my family, my family, my support worker, and I don't know. Each response option was provided a distinct numeric code and where multiple response options were selected, a new response code was created.

RQ3: What training will research participants' require to successfully engage with the NDIS?

A multi-response question set was used to ascertain what training the participants thought they would require to successfully engage with the NDIS. The participants were asked to identify whether training would be required to support their decision making across the following domains: being organised and planning time, making choices, being heard, looking after money, working on computers, talking and writing, finding people for support, and finding the right services for the right price. For each of these questions, the participants were provided the following response options: We need training, yes we can do this now, I don't know, and I don't want to do and/or I don't do this. Finally, this section ended with openended questions whereby the research participants were invited to indicate what other types of support they might want in the future.

\section{Data Analysis}

Templates were designed to ensure accuracy in documenting the interview data and depth in eliciting research participants' responses. First, the researchers completed the 'Interview Protocol Survey' (as described above) during the interview. Second, after the interview, the 
researchers transcribed the research participants' longer responses to the more narrative interview questions into a separate template, 'Qualitative Responses Notes'. These notes assisted with the qualitative data analysis outlined in this article.

All quantitative data was analysed using the Statistical Package for the Social Sciences (SPSS.Version 19.0). Descriptive statistics were tabulated for all quantitative data. The longer, more narrative interview responses in the 'Qualitative Responses Notes' were collated, and analysed for emergent, recurrent themes and unusual or exceptional responses. That is, an inductive approach was undertaken and the qualitative responses were analysed for both thematic patterns and thematic disruptions. Two independent researchers then confirmed this narrative content analysis.

\section{Findings}

In this section, the Research Participant Information section provides the demographic and background information for the sample. Findings are then presented for each research question.

\section{Research Participant Information}

People with disability were aged between 25 and 63 years (average age of 46 years) and reasonably gender matched ( $44 \%$ females, $56 \%$ males). They had used the organisation's services for an average of 24 years (ranging from 3 to 52 years). They used a range of the organisation's services including training and employment services, accommodation support, respite, and learning and lifestyle support. Some people used more than one service: $22.9 \%$ of people who used the organisation's accommodation services also used the organisation's employment support services. Additionally, 69 of 70 people with disability completed the interview with the aid of a support person. Support people included (with percentage in brackets): parents and/or guardians (48.6), organisation staff (22.9), a sibling (7.1), or multiple persons (7.1). Finally, 69 research participants were diagnosed as having an intellectual disability while one person was diagnosed as having an acquired brain injury. Analysis of EuroQoL 5D UK survey data found that compared to age norms from a United Kingdom population sample (Cheung et al. 2009), the proportion of people with disability in this study with some or many barriers on selected sub-scales was higher than would be expected for people within any age group between 18 and 65 years. Most people with disability (72\%) reported no barriers across several domains, with $28 \%$ having some or many problems overall. Most people with disability (76\%) also claimed they experienced no 
barriers concerning their self-care. However, inconsistencies arose between responses in this part of the survey and their responses to later questions about to their expressed needs for the future. Significantly, almost half of the study sample (46\%) self-reported some or extreme difficulty communicating with others. Although not representative of the NDIS population, this latter finding has profound implications for NDIS assessment of need, as well as selfdirection initiatives.

\section{RQ1: What do research participants know about the NDIS?}

Research participants were generally unaware, confused and/or held mixed expectations about the NDIS. Over $50 \%$ of research participants reported that they 'didn't know much about the NDIS', 'not really sure what it is and how it will affect me', 'don't know anything about it', 'never heard of it', 'have heard of it but don't know much about it', and 'I don't really keep up with the NDIS'. Even when research participants had attended a NDIS information seminar, they reported a lack of NDIS understanding. Several research participants who attended more than one NDIS information seminar reported being confused about what the NDIS would mean for the health and social support that is currently received.

Several research participants seemed to believe that they had a choice of opting into the NDIS or staying with their current disability service provider. They perceived their disability service provider as an entity standing alongside the NDIS, rather than as one of many national and local service providers competing for services within the new NDIS system. Other concerns were raised about their NDIS eligibility, 'how it was going to work' for their family, and how individual differences in people's situations would be taken into account. One parent reported

'we have been to two seminars and the presenters were like TV characters portraying the perfect situation. The examples they provided could not work in all situations. It depends on the child's capabilities. We want [our adult son or daughter] to be happy and safe while we are alive and after we are gone'.

Other parents who were actively informing themselves about the NDIS expressed their openness to the breadth of options that might be available for their son or daughter. Some parents had already drafted lengthy statements in preparation for their planning and assessment interviews with the NDIA.

Parents asked whether their son or daughter would continue to receive the Disability Support Pension. Other parents resolved their ambiguity about the NDIS by stating their preference 
for the current situation: 'the devil you know is better than the devil you don't know', and 'I know enough to have concerns'. Despite attending information seminars, many parents continued to be concerned, particularly about the future living situations for their adult son or daughter.

\section{RQ2: Who do research participants intend to have organise their support under the NDIS?}

Research participants were asked to indicate who they thought would make most of the decisions about their support under the NDIS. A majority (72\%) expected that their family member (with or without legal guardianship) or friend would make most of the decisions. A smaller proportion $(15 \%)$ believed that the person with disability would make most decisions about their support in future, with or without assistance from others such as family. One person with a disability reported he did not know who was going to make the decisions about his future support. Over half of the research participants expected their family or current service provider to remain their key source of support in a variety of activities in the future under the NDIS.

As self-directed activities and individual funding arrangements are core components of the NDIS, research participants were also asked who they expected would assist them, if at all, in choosing or changing supports, keeping money, finding supports, paying supports, providing training to support staff, and providing support. Table 1 lists the support group that was identified by research participants for each event. As indicated in Table 1, most research participants suggested that their family would be responsible for activities involving the organisation of support namely, the identification, paying and training of support staff. At the same time, research participants also indicated that the current service provider would provide their health and social support. Qualitative data provided insight to this point. Parents whose sons and daughters had been longstanding service users regarded the provider as their extended 'family', and thus preferred that the current provider, provide health services.

\section{RQ3: What training will research participants require to successfully engage with the} NDIS?

Research participants were asked if they or their family member or friend would need training in everyday activities such as looking after money, communicating, planning, decision making and locating cost effective services. Table 2 below includes research participant responses. 
Research participants indicated that they or their family were able to do these activities to a certain extent, but needed some or more training. The areas where the most research participants indicated needing training concerned: money management, working on computers, and finding quality, affordable services. The areas where the least proportion of research participants indicated requiring training concerned: being heard and finding people for support or friendship.

Finally, an open-ended question was included to ascertain the areas where research participants would require support in the future. Research participants generally expressed modest views about the support they would want in the future. While some of these related to training and skills development, others were about age-appropriate ideals and milestones. Research participants spoke about wanting 'more company/friendship', to 'find a job' or different job, to 'study', to improve their 'communication skills and use of a computer', 'help with public transport', 'to learn to catch a bus again', to 'learn to use a flexi-teller', 'going shopping more', 'to learn how to use my mobile phone properly', 'to manage my health and care myself', 'financial skills', 'help getting to mum's [house]', 'an apartment with my boyfriend', 'be involved in performing arts', and to 'own a car'.

\section{Discussion}

Within this study, people with a disability and their families receiving health and social support from a disability service provider in South-East Queensland provided insight into their knowledge of the NDIS, who would organise their support under the NDIS, and training that they require to successfully engage with the NDIS. The following sections discuss the research findings, and present the implications for policy and practice.

\section{National Disability Insurance Scheme Knowledge}

Research participants within this study generally had a limited understanding of the NDIS and what it would mean for the organisation of the future health and social support of people with disability. Even research participants who had attended information seminars indicated that they did not obtain much information about the impact of the NDIS on their service provision. The lack of participant understanding about the self-directed approach to disability support is a phenomenon which has been identified in global research about self-directed policies for people with disability (see for example, F. Mitchell [2012, 2014a, 2012b], Dew et al. [2013], and Lakhani, McDonald, and Zeeman [2016] for information about the lack of participant understanding of self-directed approaches). 
In Australia, participants' limited understanding about the NDIS has been identified in research investigating future participants' perspectives of the NDIS. For example, Howard et al. (2015) explored the perspectives of parents and care givers of children with disability about the NDIS. Participants were located within the Hunter Region, New South Wales, where a trial site was set to be established. Similar to participants included within this study, Howard et al's research found that many participants 'had very low-level knowledge about the NDIS and changes that may result for their child's support' (pg. 1371). Similar to this study, $50 \%$ of their sample indicated having no knowledge of the NDIS and when NDIS specific information was provided, it did not provide adequate information for participants to understand the NDIS. The findings from this study and Howard et al. (2015) provide evidence that individuals are under-informed about the NDIS.

This study adds to a growing body of knowledge concerning the understanding that people with disability have about self-directed programs. As this is an understudied area, a definitive understanding of the information needs of people with disability and guardian(s), family member(s) and/or carer(s) about the NDIS is not yet clear. Nevertheless, it is reasonable to expect that current and future NDIS participants would benefit from additional sources of information and knowledge sharing opportunities about the NDIS, including the organisation of health and social supports under the NDIS. Such knowledge sharing may contribute to people with disability and guardian(s), family member(s) and/or carer(s) having a higher level of preparation for engaging with the NDIS, and potentially result in people with disability receiving services that meet their health and social needs.

\section{Providers of support}

Research participants generally indicated that the person with disability, together with their family, would organise their supports under the NDIS, and that their current service provider would provide those supports. The current service provider was indicated as ideal by research participants due to their longstanding relationships. The service provider may have also been indicated as ideal as the research participants may have been more likely to have a favourable perspective of the service provider. Nevertheless, these findings are supported by global research investigating participant perspectives of self-directed models (see reviews by Lakhani, McDonald, and Zeeman [2016], and Sims and Cabrita Gulyurlu [2014]). Studies in the area have highlighted that family - particularly parents and guardians - typically make most of the financial and support decisions about service users' care (Gross et al., 2013). In regard to support provision, a systematic review by the authors (see Lakhani, McDonald, and 
Zeeman [2016] systematic review) found that service-users hire providers based on familiarity with the provider (Gross et al. 2013; Timberlake et al. 2014), provider experience, being comfortable with the provider (Heller et al. 2012), and experience working with the provider (W. Mitchell 2012b, F. Mitchell 2014a, 2014b; Timberlake et al. 2014).

In this study, research participants were familiar and comfortable with the service provider. Many also had a longstanding history in receiving services and supports from their provider, thus informing their decision to continue with their current service provider. While studies exploring participant engagement with self-directed disability health and support programs outside Australia have found similar results, to the knowledge of the authors, no study has identified the factors that people with disability and their guardian(s), family member(s) and/or carer(s) in Australia will use to inform their selection of service provider(s) under the NDIS. This is the first such Australian study to do so, and as a result, these findings add new knowledge concerning participant engagement with the NDIS, particularly the factors that inform participant and family member service provider selection under the NDIS. It is expected that such information about the role of familiarity, comfortableness and longstanding client-provider relationships will improve the delivery and practice of service providers.

\section{Preparation for the National Disability Insurance Scheme}

A large proportion of research participants indicated that they would require training in budgeting and identifying the right services for the right price. These important tasks have been identified by research globally. For example, budgeting and hiring support have been identified as areas which may prevent participants' successful participation within selfdirected health and social support programs (Sims and Cabrita Gulyurlu, 2014; Lakhani, McDonald, and Zeeman 2016). W. Mitchell (2012b) also evaluated the impact of a selfdirected program for disability health and social services for young people in the United Kingdom. Barriers to successful budgeting included the potential misuse of support funding, and difficulties faced when navigating a complex funding environment. On the issue of hiring support staff, participants' interviewed as a part of Junne and Huber's (2014) study indicated that self-directed approaches may result in participants hiring support staff that lack adequate training and qualifications, and that this may result in subpar health and social support services. Findings from this study add to the knowledge base confirming budgeting and hiring as domains where participants will require support to successfully engage with selfdirected policies. 
This Australian study also adds new knowledge about the training needs of people to ensure their successful engagement with the NDIS. Such information is timely and important given that the NDIS is currently being rolled out in parts of Australia, including Queensland. This finding also has important implications for disability support providers, and third party financial planners working within the NDIS context. The ability for such groups to provide budgeting services for NDIS participants can contribute towards participants' successful engagement with the NDIS, and potentially ensure the receipt of optimal health services for people with disability.

\section{Limitations}

This study has limitations to consider when interpreting the findings. This study aimed to ascertain how people with disability intend to engage with the NDIS and given the study design it is not possible to inference how they will actually engage with the NDIS. In addition, this study was limited to people with disability who were receiving support from a single provider working in South-East Queensland. People with disability receiving services from the provider were approached and a subset who volunteered to participate included. It is possible that those who participated held the service provider in high regard and as a result decided to participate. Furthermore, those who participated generally had a longstanding history with the provider (on average 24 years). Consequently, given the characteristics of the participant sample, they potentially responded more favourably to questions which aimed to ascertain their perspectives of the service provider. Thus, the findings should be generalised against participants with a similar history.

A high proportion (98.5\%) of study participants had an intellectual disability. This reflects the demographics of the potential sample (of whom $84.7 \%$ had an intellectual disability). As such, the findings reflect the perspectives of people who have an intellectual disability. Accordingly, the findings do not inevitably provide insight into the experiences and perspectives of people with physical disability about the NDIS, nor how they intend to organise their supports under the NDIS.

While this survey sought to include the perspective of potential NDIS service users, for 69 of 70 people with disability, a guardian, family member and/or carer supported their communication and/or provided a response that they felt best reflected the response that the participant would have provided. This method was employed as in most cases, a considerable communication gap between the interviewer and the participant receiving services from the 
support organisation existed, which was not remedied by the communication tool. This communication gap was ameliorated by the guardian, family member and/or carer, and as a result they asked to attend the interview to support the communication and/or provide responses. This method was viewed as adequate, as people who are familiar and have the strongest knowledge of people with disability are often the best to ensure that their perspectives are conveyed (Lakhani et al. 2017; Antonsson, Åström, Lundström, and Graneheim 2013; Healy and Noonan Walsh 2007). As such, generalisations made from this research should recognise that the opinions of family were included as a representation for the perspectives of NDIS future participants. In this regard, while there is the potential that representatives may have differing views from the people with disability who they organise supports for (as identified by Mitchell 2012a), it is expected, that for the most part, guardians and family members provided a response which strongly reflected that of the participant.

\section{Policy Implications}

This study has important implications for the policies and practices of the NDIA and service providers. This study found that prospective NDIS participants with intellectual and cognitive disabilities and their families had limited understanding of the NDIS. It is expected that targeted NDIS information delivered in an accessible way would positively influence the participants' knowledge of the NDIS. Delivering information via effective, customised communication support tools, and longer-than-average face-to-face consultations may better inform participants. In this respect, taking the adequate time, over numerous occasions to explain issues is important as it provides potential participants the time to develop educated queries and have their queries answered (McDonald, Lakhani, and Zeeman 2016).

Consistent with a recent evaluation of the NDIS in five trial sites, this study identified that participants would benefit from support in engaging with the NDIS (Mavromaras, Moskos, and Mahuteau 2016). It is expected that in order for the NDIS to meet the objectives, this support need be longstanding (Kendrick, Ward and Chenoweth 2017). There is also potential for peer-support models where people with disability and experience engaging with the NDIS provide guidance and support to those engaging with the NDIS to support participants. Such models have proven effective to support information sharing among people with severe mental illness (Chinman et al. 2014), and increased the overall wellbeing of people with psychiatric disability (Walker and Bryant 2013). They have also proven to support people with spinal cord injury adjust to life after injury and reduce medical complications that they may experience (Ljunberg, Kroll, Libin, and Gordon 2011). Given the effectiveness of such 
programs, it is worthwhile to test the effectiveness of the peer-support approach among NDIS participants.

This study clarified that the development of every-day money management skills is a pressing need among future participants, and a support opportunity to service providers for existing and future clients. Although the Public Trustee has a significant role in determining major lifestyle expenses for many people with disability, people with intellectual and cognitive disabilities need to be skilled in basic financial literacy and cash management (such as using an automatic teller machine) for their everyday needs. For people with disability or their families self-directing their services, financial assistance in acquitting support services, i.e., recruiting, changing and paying for support, is a key concern and related area for skill development and/or assistance. (See Robinson, Fisher and Gendera [2016] for a discussion about different types of flexible, person-centred planning and case management approaches to self-directed support for people with disability and their families). Thus advocates who are experts in engaging with the NDIS will be essential to support the successful participation of some families.

The findings from this study also support the notion that aligning self-directed support processes with social model of disability frameworks can encourage successful participant engagement with self-directed policies. There are diverse competing understandings of the social model of disability, however they all distinguish impairment as a physical and/or cognitive condition while disability as the loss of opportunities as a result of individual interaction with social, economic and/or physical environments (Burchardt, 2004; People with Disability Australia [PWDA], 2017). The model emphasises that environments must change to ensure that people with impairment have equal opportunity to participate (PWDA, 2017). In line with this perspective, the study identified that support providers can provide information sharing and training opportunities to ensure that people with disability successfully engage with the NDIS. Also aligning with the social model of disability, it is important that the environments under which these opportunities are provided, are contextualised to ensure individual agency and autonomy. This may be via the use of diverse communication methodologies (Lakhani, McDonald, and Zeeman 2016a; McDonald, Lakhani, and Zeeman 2016; Lakhani et al. 2017), or once again, by including those with disability throughout the training and/or information sharing processes (Burchardt, 2004).

\section{Conclusion}


For the most part, among potential participants globally, the aims and outcomes of selfdirected programs remain ambiguous. Similarly, the NDIS remains misunderstood for many people with intellectual and cognitive disability, and their families in Australia. The risk is that they will avoid or under-use the NDIS, resulting in no significant change or improvement in their support services or quality of life. Consequently, training and supporting prospective NDIS participants in self-direction emerges as the most significant issue to be tackled. Many of the research participants with intellectual and cognitive disabilities and their families face diverse and fundamental challenges that need to be addressed now as well as into the future in preparing for the NDIS. They need support with their health; assistive technology and home modifications; more effective and customised communication support including augmentative and alternative communication methods; more diverse and customised housing options; greater variety of meaningful work and leisure opportunities; support and training in financial literacy and management; and targeted but straightforward NDIS information so that they can engage in the NDIS processes and service planning. 


\section{References}

Antonsson, H., Åström, S., Lundström, M., \& Graneheim, U. H. (2013). Skilled interaction among professional carers in special accommodations for adult people with learning disabilities. Journal of psychiatric and mental health nursing, 20(7), 576-583.

Australian Government Productivity Commission. 2011. Disability care and support: Productivity commission inquiry report (No. 54). Melbourne, Victoria: Productivity Commission.

Bonyhady, B. 2014. "Tides of change: the NDIS and its journey to transform disability support." The Australian Journal on Psychosocial Rehabilitation, 7(1): 7-9.

Bourke, E. 2014. “The housing needs of NDIS participants.” Parity, 27(5): 10.

Burchardt*, T. (2004). Capabilities and disability: the capabilities framework and the social model of disability. Disability \& society, 19(7), 735-751.

Care and Support [Direct Payments] Regulations 2014

Cheung, K., M. Oemar, M. Oppe, and R. Rabin. 2009. EQ-5D User Guide. Euroqol group. www.euroqol.org

Chinman, M., George, P., Dougherty, R. H., Daniels, A. S., Ghose, S. S., Swift, A., \& Delphin-Rittmon, M. E. (2014). Peer support services for individuals with serious mental illnesses: assessing the evidence. Psychiatric Services, 65(4), 429-441.

Commonwealth of Australia. 2013. National Disability Insurance Scheme Act. Canberra: Australian Government.

Crozier, M., Muenchberger, H., Colley, J., \& Ehrlich, C. (2013). The disability self-direction movement: Considering the benefits and challenges for an Australian response. Australian Journal of Social Issues, 48(4), 455-472.

Dew, A., K. Bulkeley, C. Veitch, A. Bundy, M. Lincoln, J. Brentnall, G. Gallego, S. Griffiths. 2013. "Carer and service providers' experiences of individual funding models for children with a disability in rural and remote areas." Health \& Social Care in the Community, 21(4): 432-441.

Eriksson, S. 2014. "The need for self-determination and imagination: Personal budgeting and the management of disability services in Finland." Journal of Policy \& Practice in Intellectual Disabilities, 11(2): 137-148. doi: 10.1111/jppi.12079 
Espiner, D., and F.M. Hartnett. 2012. “'I felt I was in control of the meeting': facilitating planning with adults with an intellectual disability." British Journal of Learning Disabilities, 40(1): 62-70. doi: 10.1111/j.1468-3156.2011.00684.x

Foster, M., P. Henman, C. Tilse, J. Fleming, S. Allen, and R. Harrington. 2016. “'Reasonable and necessary' care: The challenge of operationalising the NDIS policy principle in allocating disability care in Australia.” Australian Journal of Social Issues, 51(1): 2746.

Grant, E., A. Chong, A. Beer, and A. Srivastava. 2014. "The NDIS, housing and Indigenous Australians living with a disability." Parity, 27(5): 25.

Gross J.M.S., L. Wallace, M. Blue-Banning, J.A. Summers, and A. Turnbull. 2013. "Examining the experiences and decisions of parents/guardians: participant directing the supports and services of adults with significant intellectualand developmental disabilities." Journal of Disability Policy Studies, 24:88-101.

Harkes M. A., M. Brown, and D. Horsburgh. 2014. "Self Directed Support and people with learning disabilities: a review of the published research evidence.” British Journal of Learning Disabilities, 42:87-101.

Healy, D., \& Noonan Walsh, P. (2007). Communication among nurses and adults with severe and profound intellectual disabilities: predicted and observed strategies. Journal of Intellectual Disabilities, 11(2), 127-141.

Heller, T., K. Arnold, L. van Heumen, E. McBride, and A. Factor. 2012. “Consumer-directed support: Impact of hiring practices on adults with ID/DD and families.” International Journal of Integrated Care, 12(9). DOI: http://doi.org/10.5334/ijic.1091.

Howard, A., T. Blakemore, L. Johnston, D.Taylor, and R. Dibley. 2015. "'I'm not really sure but I hope it's better': early thoughts of parents and carers in a regional trial site for the Australian National Disability Insurance Scheme.” Disability \& Society, 30(9): $1365-1381$.

Junne, J., and C. Huber. 2014. "The risk of users' choice: exploring the case of direct payments in German social care.” Health, Risk \& Society, 16(7-8): 631-648.

Kelly, S., and M. Sheehan. 2014. "What is a reasonable and necessary support to live in safe, appropriate affordable housing?" Parity, 27(5): 33. 
Kendrick, M., Ward, M., \& Chenoweth, L. (2017). Australia's national disability insurance scheme: looking back to shape the future. Disability \& Society, 1-18.

Lakhani, A., McDonald, D., \& Zeeman, H. (2016). Perspectives of self-direction: a systematic review of key areas contributing to service users' engagement and choicemaking in self-directed disability services and supports. Health \& social care in the community.

Ljungberg, I., Kroll, T., Libin, A., \& Gordon, S. (2011). Using peer mentoring for people with spinal cord injury to enhance self-efficacy beliefs and prevent medical complications. Journal of clinical nursing, 20(3-4), 351-358.

Lloyd, L. 2002. "Caring relationships: Looking beyond welfare categories of 'carers' and 'service users."' In Stalker, K. (Ed.), Reconceptualising work with 'carers': New directions for policy and practice, London: Jessica Kingsley: 37-55.

Mavromaras, K., Moskos, M., \& Mahuteau, S. (2016). Evaluation of the NDIS.

Mcdonald, D., Lakhani, A., \& Zeeman, H. (2016). Ethics of time: Ensuring effective relational communication with NDIS participants who have intellectual disabilities. Journal of Intellectual Disability Research, 60(7), 706.

McIntyre, M., and E. Kendall. 2013. "Family resilience and traumatic brain injury." In H. Muenchberger, E. Kendall \& J. Wright (Eds), Health and healing after traumatic brain injury: Understanding the power of family, friends, community and other support systems, Westport, CT: Praeger: 57-69.

Mitchell, F. 2014a. "Facilitators and barriers to informed choice in self-directed support for young people with disability in transition." Health \& Social Care in the Community, 23(2): 190-199.

Mitchell, F. 2014b. "Informed Choice for Disabled Young People in Transition: A Secondary Analysis of Qualitative Data." Practice, 26(4): 259-270. doi: $10.1080 / 09503153.2014 .934800$

Mitchell F. 2012. "Self-directed support and disabled youngpeople in transition (part 2)." Journal of Integrated Care, 20:223-230.

Mitchell W. 2012a. "Perspectives of Disabled Young People with Degenerative Conditions on Making Choices with Parents and Peers." Qualitative Social Work, 11: 621-643. 
Mitchell, W. 2012b. "Parents' accounts: Factors considered when deciding how far to involve their son/daughter with learning disabilities in choice-making." Children and Youth Services Review, 34(8): 1560-1569. doi: 10.1016/j.childyouth.2012.04.009

Murphy, J., and L. Cameron. 2008. "The effectiveness of Talking Mats ${ }^{\circledR}$ with people with intellectual disability.” British Journal of Learning Disabilities, 36: 232-241.

National Commission of Audit. n.d. Australian Government: http://www.ncoa.gov.au/report/appendix-vol-1/9-2-national-disability-insurancescheme.html

Ottman, G., M. Mohebbi, and A. Millicer. 2014. "Client self-assessment in community aged care: A comparative study involving older Australians and their case managers." Sage Open, 4(1): 1-10.

People with Disability Australia (2017). The Social Model of Disability. Accessed from http://www.pwd.org.au/student-section/the-social-model-of-disability.html on 3rd January 2017.

Ranasinghe, T., J. Deepa, D. White, and R. Russo. 2017. "Parents' experiences in registering with and accessing funding under the National Disability Insurance Scheme for early intervention services for children with developmental disabilities." Journal of Paediatrics and Child Health, 53(1): 26-32.

Remington-Gurney, J. 2013. "Scaffolding conversations using augmentative and alternative communication (AAC)." Journal of Social Inclusion, 4(1):45-69.

Robinson, S., K. Fisher, and S. Gendera. 2016. "Can case management contribute to effective use of small self-directed support packages for people with disabilities?” Asian Social Work and Policy Review, 10(3): 268-279. DOI: 10.1111/aswp.12095.

Russi, M. V. 2014. "NDIS and occupational therapy: Compatible in intention and purpose from the consumer perspective." Australian occupational therapy journal, 61(5): 364370.

Sims D., and S.S.C. Gulyurtlu. 2014. "A scoping review of personalisation in the UK: approaches to social work and people with learning disabilities." Health \& Social Care in the Community, 22:13-21.

Social Services and Well-being (Wales) Act 2014 
Timberlake M.T., W.N. Leutz, M.E. Warfield, and G. Chiri. 2014. "In the Driver's Seat" : parent perceptions of choice in a participant-directed Medicaid waiver program foryoung children with autism." Journal of Autism and Developmental Disorders, 44: 903-914.

Ward, M., J. Franz, and B. Adkins. 2014. Livable housing design: The voluntary provision of inclusive housing in Australia, Journal of Social Inclusion, 5(1): 43-60.

Wiesel, I., and D. Habibis. 2015. NDIS, housing assistance and choice and control for people with disability. AHURI Final Report.

Wiesel, I. 2015. "Housing for People with Intellectual Disabilities and the National Disability Insurance Scheme Reforms." Research and Practice in Intellectual and Developmental Disabilities, 2(1): 45-55. 
Table 1: Future support

\begin{tabular}{|c|c|}
\hline Domain & Highest Identified Support Group (percentage) \\
\hline $\begin{array}{l}\text { Choosing } \\
\text { help }\end{array}$ & $\begin{array}{l}\text { Me and my family (28.6) } \\
\text { My family (20.0) } \\
\text { Current service provider (10.0) } \\
\text { Me, my family and current service provider }(7.1) \\
\text { My support worker from current provider }(5.7) \\
\text { Me and my support worker from a different provider (2.9) } \\
\text { Me by myself (1.4) } \\
\text { I don't know (1.4) } \\
\text { My Family and current service provider (1.4) } \\
\text { Me and current service provider (1.4) } \\
\text { Family and I don't know } \\
\text { Doctor (1.4) } \\
\text { Current service provider and my support worker from a different provider } \\
\text { (1.4) } \\
\text { My family, support worker from a different provider and current service } \\
\text { provider (1.4) }\end{array}$ \\
\hline $\begin{array}{l}\text { Changing } \\
\text { help }\end{array}$ & $\begin{array}{l}\text { My family (22.9) } \\
\text { Me and my family (21.4) } \\
\text { Current service provider }(10.0) \\
\text { Me, my family and current service provider (4.3) } \\
\text { My family and support worker from a different provider (4.3) } \\
\text { My support worker from current provider (2.9) } \\
\text { I don't know (2.9) } \\
\text { My Family and current service provider (2.9) } \\
\text { Me and my support worker from a different provider (2.9) } \\
\text { Me and current service provider (1.4) } \\
\text { Family and I don't know } \quad(1.4) \\
\text { My family, support worker from a different provider and current service } \\
\text { provider (1.4) }\end{array}$ \\
\hline $\begin{array}{l}\text { Keeping } \\
\text { money }\end{array}$ & $\begin{array}{l}\text { My family (31.4) } \\
\text { Public Trust (17.1) } \\
\text { Me and my family (14.3) } \\
\text { Me by myself (4.3) } \\
\text { Family and I don't know } \\
\text { Me, my family and current service provider (4.3) } \\
\text { My support worker from current provider (2.9) } \\
\text { Current service provider (2.9) } \\
\text { I don't know (1.4) } \\
\text { Current service provider and Public Trustee (1.4) }\end{array}$ \\
\hline $\begin{array}{l}\text { Finding } \\
\text { support }\end{array}$ & $\begin{array}{l}\text { Current service provider (30.0) } \\
\text { My family (25.7) } \\
\text { Me and my family (8.6) } \\
\text { Me, my family and current service provider (4.3) } \\
\text { I don't know (2.9) } \\
\text { My Family and current service provider (1.4) }\end{array}$ \\
\hline
\end{tabular}


Table 2: Training required

Do you or your family member or friend have these skills (sorted descending by 'Needs Training') (numbers in percentages)

\begin{tabular}{l|rrrr}
\hline & $\begin{array}{r}\text { Need } \\
\text { Training }\end{array}$ & $\begin{array}{r}\text { Yes, we } \\
\text { can do } \\
\text { now }\end{array}$ & I don't know & $\begin{array}{r}\text { I don't want } \\
\text { to or I don't } \\
\text { do this }\end{array}$ \\
\hline Looking after Money & 40 & 20 & 10 & 4.3 \\
Working on computers & 32.9 & 17.1 & 7.1 & 14.3 \\
Finding right services for right & 31.4 & 15.7 & 22.9 & 5.7 \\
Talking and Writing & 28.6 & 24.3 & 4.3 & 5.7 \\
Planning my time & 24.3 & 35.7 & 11.4 & 4.3 \\
Making Choices & 22.9 & 35.7 & 10 & 2.9 \\
Being Heard & 18.6 & 42.9 & 11.4 & 1.4 \\
Finding people for support or & 18.6 & 37.1 & 14.3 & -- \\
\hline
\end{tabular}

